\title{
Analysis of advanced lung cancer patients diagnosed following emergency admission
}

\author{
Daichi Fujimoto ${ }^{1}$, Ryoko Shimizu ${ }^{1}$, Takeshi Morimoto ${ }^{2,3}$, Ryoji Kato ${ }^{1}$, Yuki Sato ${ }^{1}$, \\ Mariko Kogo ${ }^{1}$, Jiro Ito ${ }^{1}$, Shunsuke Teraoka ${ }^{1}$, Takehiro Otoshi ${ }^{1}$, Kazuma Nagata ${ }^{1}$, \\ Atsushi Nakagawa ${ }^{1}$, Kojiro Otsuka ${ }^{1}$, Nobuyuki Katakami ${ }^{1}$ and Keisuke Tomii ${ }^{1}$
}

\footnotetext{
Affiliations:

${ }^{1}$ Dept of Respiratory Medicine, Kobe City Medical Center General Hospital, Kobe, Japan.

${ }^{2}$ Clinical Research Center, Kobe City Medical Center General Hospital, Kobe, Japan.

${ }^{3}$ Division of General Internal Medicine, Hyogo College of Medicine, Nishinomiya, Japan.
}

\section{Correspondence:}

Daichi Fujimoto, Dept of Respiratory Medicine, Kobe City Medical Center General Hospital, 2 MinatojimaMinamimachi, Chuo-ku, Kobe 650-0047, Japan. E-mail: daichianzendyahoo.co.jp

ABSTRACT Data on prognosis and predictors of overall survival in advanced lung cancer patients diagnosed following emergency admission (DFEA) are currently lacking.

We retrospectively analysed data from 771 patients with advanced nonsmall cell lung cancer between April 2004 and April 2012.

Of the 771 patients, 103 (13\%) were DFEA. DFEA was not an independent predictor of overall survival by multivariate Cox proportional hazard models, whereas good performance status (PS), epidermal growth factor receptor gene mutation, stage IIIB, adenocarcinoma and chemotherapy were independent predictors of overall survival (hazard ratio (95\% CI) 0.36 (0.29-0.44), $\mathrm{p}<0.001 ; 0.49$ (0.38-0.63), $\mathrm{p}<0.001 ; 0.64$ (0.51$0.80), \mathrm{p}<0.001 ; 0.81$ (0.67-0.99), $\mathrm{p}=0.044$; and 0.40 (0.31-0.52), $\mathrm{p}<0.001$, respectively). Good PS just prior to opting for chemotherapy, but not at emergency admission, was a good independent predictor of overall survival in DFEA patients (hazard ratio (95\% CI) $0.26(0.12-0.55)$; $\mathrm{p}<0.001)$.

DFEA is relatively common. DFEA and PS at emergency admission were not independent predictors of overall survival, but good PS just prior to opting for chemotherapy was an independent predictor of longer overall survival. Efforts to improve patient PS after admission should be considered vital in such circumstances.

@ERSpublications

Efforts to improve performance status after admission are vital in patients diagnosed following emergency admission http://ow.ly/C3BDQ

For editorial comments see Eur Respir J 2015; 45: 867-868 [DOI: 10.1183/09031936.00198814]

Received: April 102014 | Accepted after revision: Aug 292014 | First published online: Oct 162014

Support statement: This study was supported by internal funding and the Ethics Committee of Kobe City Medical Center General Hospital.

Conflict of interest: None declared.

Copyright OERS 2015 


\section{Introduction}

Lung cancer is the leading cause of cancer-related deaths worldwide $[1,2]$. Nonsmall cell lung cancer (NSCLC) accounts for $\sim 80 \%$ of lung cancers, and the majority are already unresectable and metastatic upon their initial diagnosis $[1,2]$. Some patients are admitted to hospital through the emergency department with lung cancer-associated symptoms, and this route to diagnosis, defined as the sequence of interactions between the patient and the healthcare system, has been well-studied in the UK. Indeed, about $40 \%$ of patients with lung cancer are diagnosed following an emergency admission (DFEA) to hospital, the highest percentage for any cancer, and survival is particularly poor in this group, most of whom have advanced disease [3]. DFEA is more common in women, and more likely with increasing age and deprivation $[4,5]$. However, there are relatively few data on prognosis and prognostic factors in patients with advanced lung cancer DFEA. Additionally, the route to diagnosis differs among countries.

The clinical features of DFEA patients are heterogeneous. Although previous studies found that performance status (PS) was poorer in DFEA patients compared with non-DFEA patients, the reasons for emergency admission and the cause of poor PS vary [6]. Furthermore, we have experienced some DFEA patients whose PS was improved following non-chemotherapeutic supportive care after emergency admission, such as radiation therapy, drainage of fluid or antibiotics [7-9]. Understanding the demographics and clinical features of patients who present as an emergency is, therefore, important in facilitating the appropriate interventions.

The aim of this study was to obtain a detailed understanding of the characteristics, needs, experiences and outcomes of patients with lung cancer who were DFEA and to identify areas with scope for improving the care provided to this group of patients and their carers.

\section{Patients and methods}

\section{Patients}

The Kobe City Medical Center General Hospital (Kobe, Japan) is a 900-bed, tertiary referral centre, which plays a central role in treating emergency patients from the surrounding area. We retrospectively analysed patients diagnosed with advanced (stage IIIB or IV) NSCLC at this hospital between April 2004 and April 2012 (fig. 1). Patients who reported never having smoked were defined as never-smokers, those who had smoked within 1 year of the diagnosis were categorised as current smokers and the rest were considered to be former smokers. All patients were classified on the basis of clinical stage according to the 7th edition TNM (tumour, node, metastasis) classification [10]. DFEA patients were defined as those without a lung cancer diagnosis before admission to our hospital via the emergency department and who presented directly (non-referral) to hospital with symptoms associated with lung cancer as their main complaint. We defined the patients referred to our hospital from other institutions because of suspected of lung cancer, as well as those admitted to our hospital because of symptoms not associated with lung cancer, as non-DFEA. The reason for emergency admission was evaluated by at least two pulmonologists using case

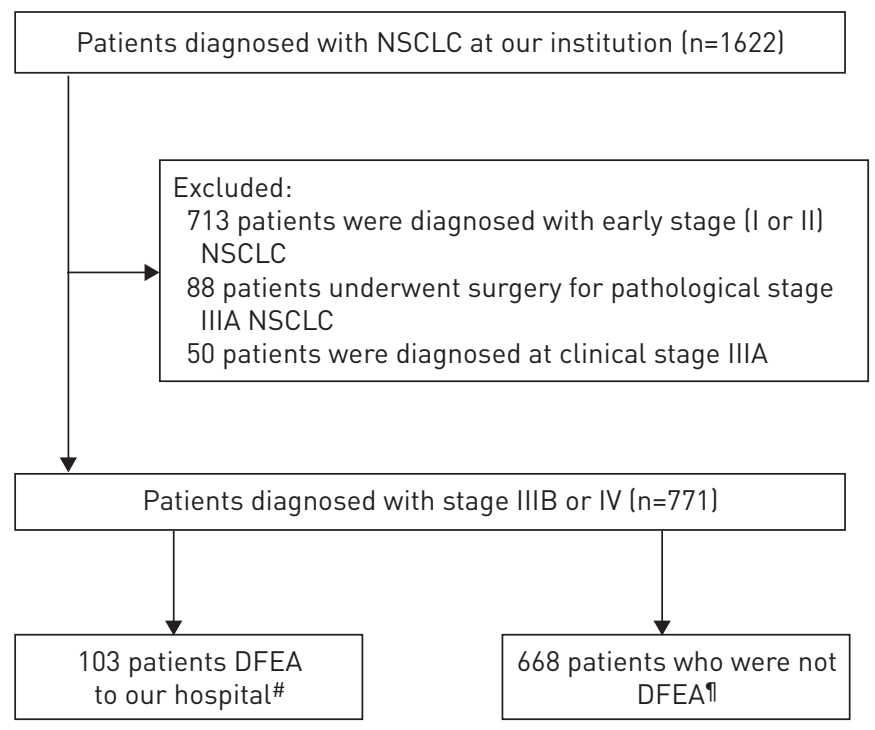

FIGURE 1 Patient selection and exclusion criteria. NSCLC: nonsmall cell lung cancer; DFEA: diagnosed following an emergency admission. " : 21 patients were lost to follow-up and were censored, and 73 events occurred; ': 121 patients were lost to follow-up and were censored, and 493 events occurred. 
and radiographic records. Overall survival was measured as the period from the diagnosis of lung cancer until death from any cause or the end of the follow-up. The Eastern Cooperative Oncology Group (ECOG) PS of DFEA patients was evaluated at emergency admission and just prior to opting for chemotherapy, and the PS of non-DFEA patients was evaluated just prior to opting for chemotherapy. "Just prior to opting for chemotherapy" was defined as the time when the decision to start chemotherapy or not was made. Improvement in PS was defined as either a change from a PS of 2 at emergency admission to a PS of 0 or 1 just prior to opting for chemotherapy, or from a PS of 3 or 4 to a PS of 0-2 by supportive care other than systemic chemotherapy. We isolated tumour DNA from various specimens and analysed epidermal growth factor receptor gene (EGFR) mutation status at exons 18-21 using the peptide nucleic acid-locked nucleic acid PCR clamp method, as described previously [11]. All chart reviews were approved by the Ethics Committee of Kobe City Medical Center General Hospital.

\section{Statistical analysis}

Continuous variables were analysed using t-tests. Dichotomous variables were analysed using Chi-squared or Fisher's exact tests, as appropriate. The Kaplan-Meier method was used to estimate the survival outcomes and groups were compared using the log-rank test. Cox proportional hazard models were fitted to determine the associations between patient characteristics and survival outcomes. A multivariate Cox proportional hazard model was developed on all clinically important factors to determine the associations between patient characteristics and survival outcomes in all patients included in this study. When we conducted subgroup analysis on DFEA patients, a similar multivariate model was constructed on variables selected by univariate analysis $(\mathrm{p}<0.05)$ because of the small number of events. The results are expressed as hazard ratios (HRs) with $95 \%$ confidence intervals. A p-value of $<0.05$ was considered to indicate statistical significance. We conducted statistical analyses using JMP 9 software (SAS Institute, Cary, NC, USA).

\section{Results}

\section{Patient characteristics}

A total of 771 patients with advanced NSCLC were included in the study, of whom 103 (13\%) were DFEA. Patient characteristics and comparisons between DFEA and non-DFEA patients are summarised in table 1. Most patients (67\%) had a PS of 0 or 1 just prior to opting for chemotherapy and adenocarcinoma (69\%). EGFR mutations were investigated in 368 (48\%) patients. Of these, EGFR mutations were detected in 128 (35\%) patients. In the DFEA patients, the main reasons for emergency admission were brain metastasis symptoms (23\%), pleural effusion (16\%) and pneumonia (15\%).

Comparison of the clinical profiles of DFEA and non-DFEA patients showed that DFEA patients included significantly lower proportions of patients with good PS (33 out of 103 versus 483 out of 668, respectively; $\mathrm{p}<0.001$ ) and patients who received chemotherapy, including definitive chemoradiotherapy (65 out of 103 versus 586 out of 688 , respectively; $\mathrm{p}<0.001)$.

We also investigated the reasons of not offering specific lung cancer treatment, except for poor PS. Of the 58 DFEA and 596 non-DFEA patients with PS 0-2, 5 (9\%) and 48 (8\%) patients did not receive chemotherapy because of patient preference.

\section{Analyses of overall survival time}

Overall survival time is summarised in table 2 and figure 2. At the time of analysis, the median (95\% CI) overall survival was 15.4 (14.1-17.0) months. Findings from univariate analyses using Cox proportional hazards models of the influence of clinical characteristics on survival outcomes indicated that longer overall survival was significantly associated with younger age, female sex, never-smoker, good PS, mutated EGFR, stage IIIB, adenocarcinoma, chemotherapy and non-DFEA. Multivariate analysis identified good PS, mutated EGFR, stage IIIB, adenocarcinoma and chemotherapy as good independent predictors of overall survival (HR (95\% CI) 0.36 (0.29-0.44), $\mathrm{p}<0.001 ; 0.49$ (0.38-0.63), $\mathrm{p}<0.001 ; 0.64$ (0.51-0.80), $\mathrm{p}<0.001 ; 0.81$ (0.67-0.99), $\mathrm{p}=0.044$; and 0.40 (0.31-0.52), $\mathrm{p}<0.001$, respectively).

\section{Analyses of overall survival time in DFEA patients}

Subgroup analyses of overall survival time in DFEA patients are summarised in table 3. Findings from univariate analyses using Cox proportional hazards models indicated that longer overall survival was significantly associated with good PS at emergency admission, good PS just prior to opting for chemotherapy, adenocarcinoma and chemotherapy. Multivariate analysis identified good PS just prior to opting for chemotherapy as a good independent predictor of overall survival (HR 0.26 (95\% CI 0.12-0.55); p<0.001).

\section{Analyses of the predictive factor for improved PS in DFEA patients}

We investigated the predictive factors for PS improvement in DFEA patients (table 4). 95 DFEA patients who had PS of 2-4 were analysed. PS improvements were significantly more frequent in younger patients 


\begin{tabular}{|c|c|c|c|c|}
\hline Age years mean $\pm s D$ & $68.9 \pm 11.1$ & $67.8 \pm 14.1$ & $69.0 \pm 10.6$ & 0.287 \\
\hline Sex & & & & 0.144 \\
\hline Male & $520(66)$ & $63(61)$ & $457(68)$ & \\
\hline Female & $251(34)$ & 40 (39) & $211(32)$ & \\
\hline Never-smoker & $218(28)$ & 23 (22) & 195 (29) & \\
\hline Current or former smoker & $553(72)$ & $80(78)$ & $473(71)$ & \\
\hline Histology & & & & $0.385^{\pi}$ \\
\hline Adenocarcinoma & 533 (69) & 75 (73) & 458 (69) & \\
\hline Squamous & $198(26)$ & $18(17)$ & $180(27)$ & \\
\hline NSCLC-NOS & $38(5)$ & $10(10)$ & $28(4)$ & \\
\hline Other & $2(0)$ & $0(0)$ & $2(0)$ & \\
\hline ECOG PS just prior to opting for chemotherapy & & & & $<0.001^{\uparrow}$ \\
\hline 0 or 1 & $516(67)$ & 33 (32) & 483 (72) & \\
\hline 2 & 138 (18) & $25(24)$ & $113(17)$ & \\
\hline 3 or 4 & $117(15)$ & $45(44)$ & $72(11)$ & \\
\hline Stage & & & & 0.281 \\
\hline IIIB & $158(20)$ & $17(17)$ & $141(21)$ & \\
\hline IV & $613(80)$ & $86(83)$ & $527(79)$ & \\
\hline EGFR status & & & & $0.359^{\pi}$ \\
\hline Mutated & $128(17)$ & $14(14)$ & 115 (17) & \\
\hline Wild-type & $240(31)$ & $28(27)$ & 212 (32) & \\
\hline Not investigated & 403 (52) & $61(59)$ & $341(51)$ & \\
\hline Treatment & & & & $<0.001^{\pi}$ \\
\hline \multicolumn{5}{|l|}{ Reasons for emergency admission } \\
\hline Pleural effusion & & $17(16)$ & & \\
\hline Pneumonia & & $15(15)$ & & \\
\hline Carcinomatous lymphangiomatosis & & $9(9)$ & & \\
\hline Pericardial effusion & & $8(8)$ & & \\
\hline Effusion only $\mathrm{n}$ & & 4 & & \\
\hline Tamponade $\mathrm{n}$ & & 4 & & \\
\hline Pain & & $6(6)$ & & \\
\hline Other & & $24(23)$ & & \\
\hline
\end{tabular}

Data are presented as $\mathrm{n}(\%)$, unless otherwise stated. DFEA: diagnosed following an emergency admission; NSCLC: nonsmall cell lung cancer; NOS: not otherwise specified; ECOG: Eastern Cooperative Oncology Group; PS: performance status; EGFR: epidermal growth factor receptor gene. ": main symptom; ": patients with adenocarcinoma and non-adenocarcinoma, patients with ECOG PS of 0 or 1 and $2-4$, patients with mutated and wild-type or not investigated EGFR, and patients with chemotherapy or chemoradiotherapy and no chemotherapy, were compared using Chi-squared tests.

compared with older patients ( 28 out of 59 versus 9 out of 36, respectively; $\mathrm{p}=0.033$ ), patients with a PS of 2 compared with those with a PS of 3-4 (16 out of 29 versus 21 out of 66 , respectively; $\mathrm{p}=0.041$ ) and in patients admitted because of pleural effusion compared with those without pleural effusion (12 out of 16 versus 25 out of 79, respectively; $\mathrm{p}=0.002$ ). The median interval between the emergency admission and the diagnosis was 3 days (interquartile range: 1-8 days), and the median interval between the diagnosis and the time when the decision to start chemotherapy or not was made (just prior to opting for chemotherapy) was 16 days (interquartile range: $8-29$ days).

We also investigated the patients who were admitted because of symptoms associated with brain metastases as it was a main reason for emergency admission. Of these 24 patients, 12 patients underwent radiotherapy, 
TABLE 2 Analyses of overall survival time

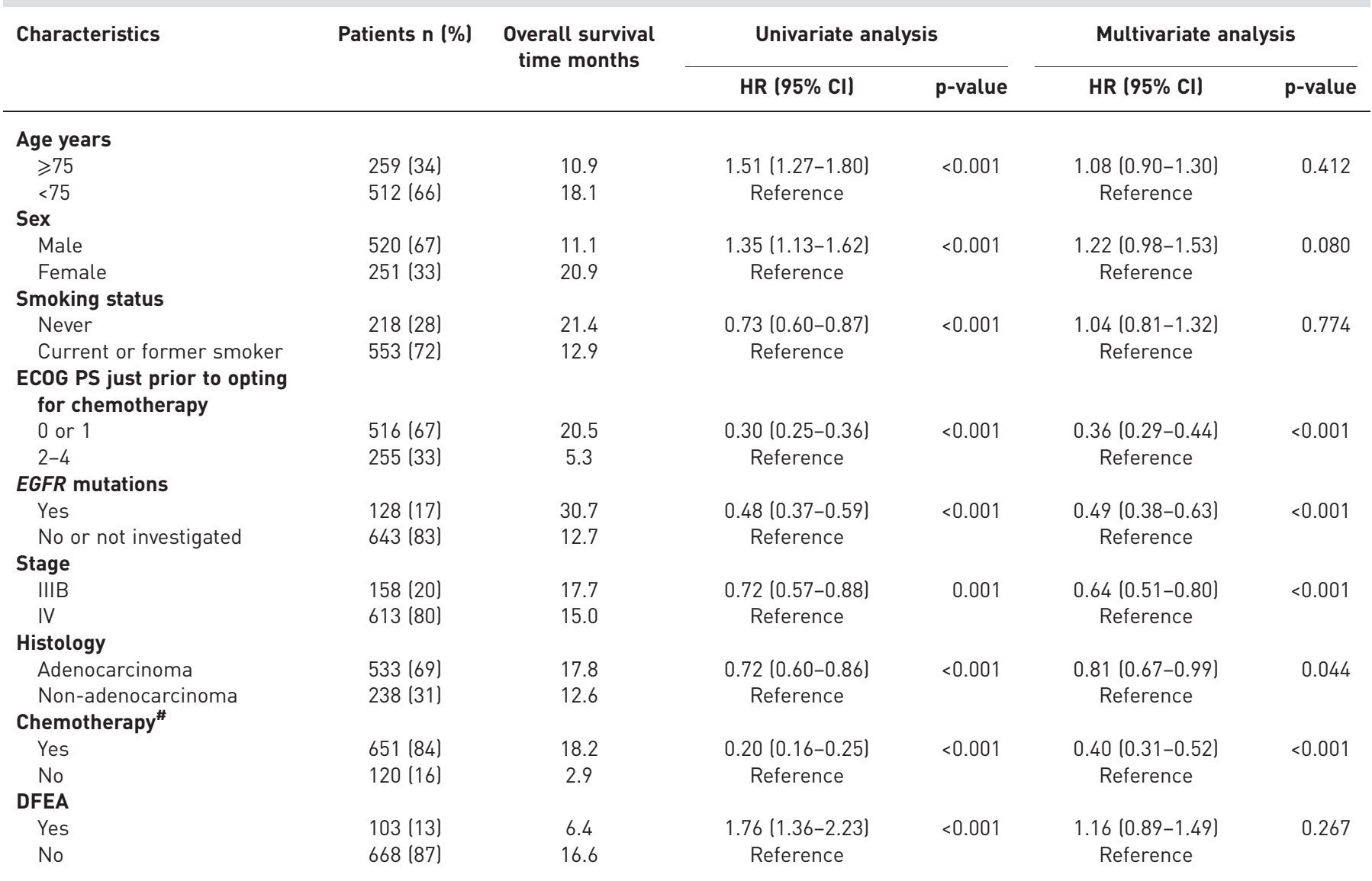

HR: hazard ratio; ECOG: Eastern Cooperative Oncology Group; PS: performance status; EGFR: epidermal growth factor receptor gene; DFEA: diagnosed following an emergency admission. " : Chemotherapy, including definitive chemoradiotherapy.

three underwent operation, six underwent operation and radiotherapy, and three received no therapy. There were six (50\%), one (33\%), five (83\%) and $0(0 \%)$ patients who presented improved PS, respectively.

\section{Discussion}

To the best of our knowledge, this study provides the first report of the characteristics, prognosis and predictors of overall survival in DFEA patients with advanced lung cancer.

Our results indicated that $13 \%$ of advanced NSCLC patients were DFEA. This is lower than in previous studies from the UK, which reported that $20-40 \%$ of advanced lung cancer patients were DFEA [3,6]. The reason for this discrepancy is unclear. One possible explanation may be the difference in the definition of DFEA; we used a narrow definition of DFEA in the current study and considered other non-elective referrals, such as emergency referrals to pulmonologists, as non-DFEA. Another possible explanation may be a difference in the accessibility of respiratory specialists; the route to specialists in the UK is generally through a general practitioner, while patients in Japan may see specialists directly, leading to shorter diagnostic delays and less cancer progression during the interval. From these points, quality management in lung cancer care might be very important to reduce the number of DFEA patients, as reported previously in European countries [12].

In this study, the main reasons for emergency admissions were symptoms of brain metastasis, pleural effusion and pneumonia. Previous studies reported that patients who had already been diagnosed with lung cancer mainly visited the emergency department with respiratory or neuropsychiatric symptoms, fever and pain [13-15]. Another study reported that the most frequent cause of intensive care unit (ICU) admission in cancer patients was respiratory failure [16]. The reasons for emergency department visits may, thus, be similar between patients before and after the diagnosis of lung cancer.

In this study, we adapted overall survival as the criterion for predicting treatment efficacy, because it has been reported that overall survival is still the best criterion for this purpose [17]. Overall survival was 

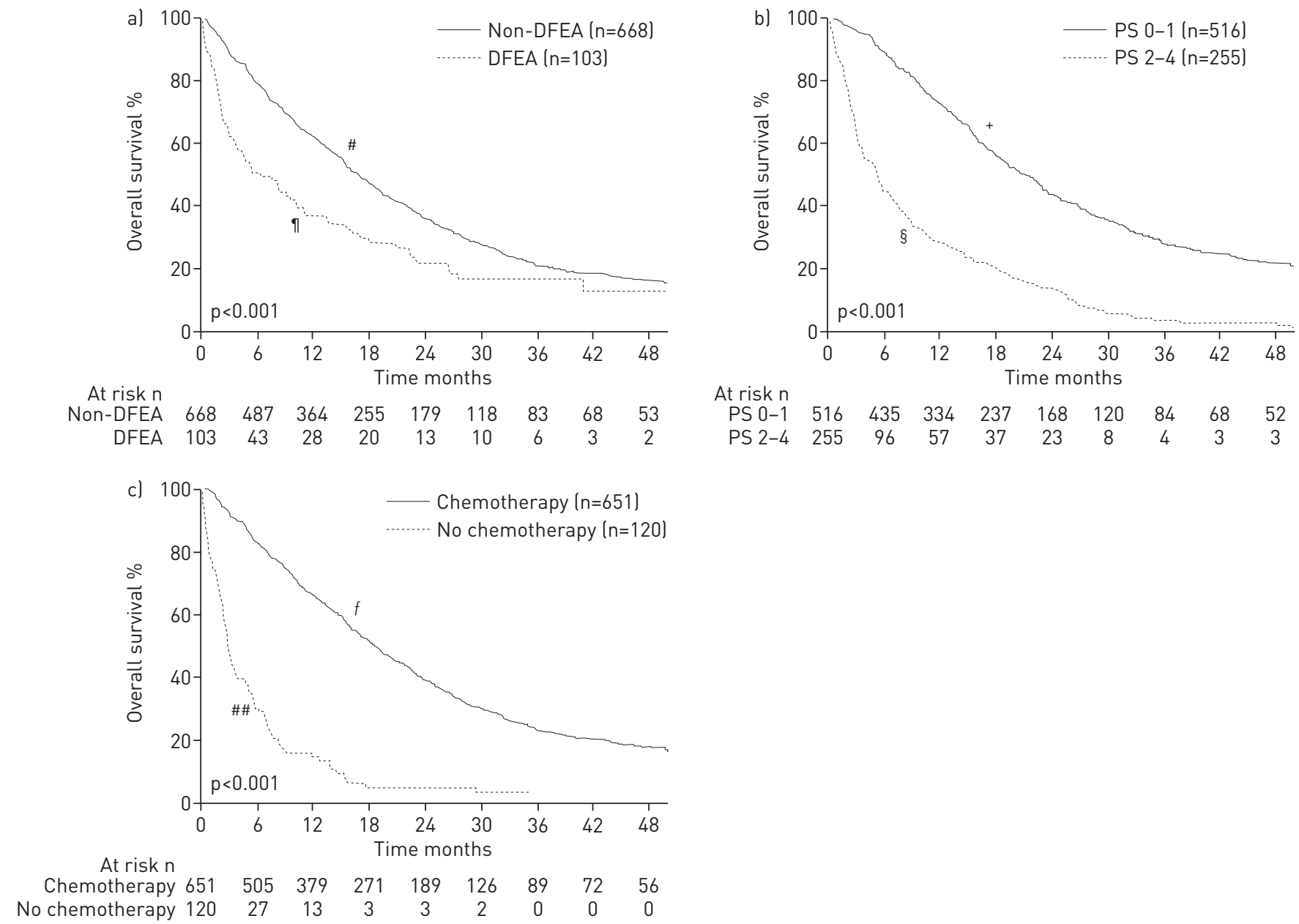

FIGURE 2 Kaplan-Meier survival curves of overall survival in a) diagnosed following an emergency admission (DFEA) and non-DFEA patients, b) in patients with performance status (PS) $0-1$ and 2-4 and c) in patients who did or did not receive chemotherapy. *: median survival 16.6 months (493 events in 668 patients); 9 : median survival 6.4 months (73 events in 103 patients); ${ }^{+}:$median survival 20.5 months ( 355 events in 516 patients); ${ }^{5}$ : median survival 5.3 months (211 events in 255 patients); ${ }^{f}$ : median survival 18.2 months (471 events in 651 patients); ${ }^{\# \#}$ : median survival 2.9 months (95 events in 120 patients).

significantly shorter in DFEA compared with non-DFEA patients, as reported in previous studies $[6,18]$. However, DFEA was not an independent predictor of overall survival according to multivariate analysis, while PS just prior to opting for chemotherapy, mutated EGFR, stage IV, adenocarcinoma histology and chemotherapy were independent predictors. Additionally, subgroup analyses of DFEA patients indicated that good PS just prior to opting for chemotherapy, but not at emergency admission, was the only independent predictor of longer overall survival. Together with the characteristics of DFEA patients, these findings suggest that shorter overall survival in DFEA patients may be associated with their poor PS, and efforts to improve patient PS after emergency admission should, thus, be considered vital in such circumstances.

In this study, a small number of patients (10 (10\%) out of 103) were admitted to the ICU. Although it is possible that most DFEA patients did not require ICU admission, this result may be a product of the triage policies at our hospital. Almost all the patients admitted at the emergency room were hospitalised in an intermediate care unit, which is an ICU step-down unit for patients who need a higher level of care, such as vasopressor infusions or noninvasive mechanical ventilation. Patients who required more intensive care, including intubation, were admitted to the ICU at our hospital. Thus, almost all the patients received intensive care soon after the emergency admission. In this study, 15 (15\%) and seven (7\%) DFEA patients required mechanical ventilation and vasopressors, respectively. The prognoses of these 22 patients were poor (median (95\% CI) overall survival: 3.1 (1.7-10.8) months). Similar results were obtained in previous studies for patients requiring ICU admission [19-24]. However, five (23\%) patients who required mechanical ventilation or vasopressor treatment survived for a period longer than 6 months. In a recent report, the prognosis of cancer patients with good PS requiring ventilatory support was better [25]. In addition, another study reported that ICU management may be appropriate, especially in patients with 
TABLE 3 Analyses of overall survival time in DFEA patients

\begin{tabular}{|c|c|c|c|c|c|c|}
\hline \multirow[t]{2}{*}{ Characteristics } & \multirow[t]{2}{*}{ Patients n $(\%)^{\#}$} & \multirow[t]{2}{*}{ Overall survival time months } & \multicolumn{2}{|c|}{ Univariate } & \multicolumn{2}{|c|}{ Multivariate } \\
\hline & & & $\operatorname{HR}(95 \% \mathrm{CI})$ & p-value & $\operatorname{HR}(95 \% \mathrm{CI})$ & p-value \\
\hline \multicolumn{7}{|l|}{ Age } \\
\hline$\geqslant 75$ years & $37(36)$ & 3.3 & $1.52(0.94-2.44)$ & 0.089 & & \\
\hline$<75$ years & $66(64)$ & 8.1 & Reference & & & \\
\hline \multicolumn{7}{|l|}{ Sex } \\
\hline Male & $63(61)$ & 4.7 & $1.17(0.73-1.89)$ & 0.515 & & \\
\hline Female & $40(39)$ & 10.1 & Reference & & & \\
\hline \multicolumn{7}{|l|}{ Smoking status } \\
\hline Never & 23 (22) & 11.1 & $0.82(0.47-1.37)$ & 0.457 & & \\
\hline Current or former smoker & $80(78)$ & 3.6 & Reference & & & \\
\hline \multicolumn{7}{|c|}{ ECOG PS at emergency admission } \\
\hline $0-2$ & $37(36)$ & 16.0 & $0.46(0.27-0.74)$ & 0.002 & $0.86(0.40-1.75)$ & 0.682 \\
\hline $3-4$ & $66(64)$ & 2.9 & Reference & & Reference & \\
\hline \multicolumn{7}{|c|}{ ECOG PS just prior to opting for chemotherapy } \\
\hline $0-2$ & $58(56)$ & 13.5 & $0.20(0.12-0.34)$ & $<0.001$ & $0.26(0.12-0.55)$ & $<0.001$ \\
\hline $3-4$ & $45(44)$ & 2.1 & Reference & & Reference & \\
\hline \multicolumn{7}{|l|}{ EGFR mutations } \\
\hline Yes & $14(14)$ & 17.8 & $0.64(0.32-1.18)$ & 0.160 & & \\
\hline No or not investigated & $89(86)$ & 4.7 & Reference & & & \\
\hline \multicolumn{7}{|l|}{ Stage } \\
\hline IIIB & $17(17)$ & 17.8 & $0.54(0.24-1.06)$ & 0.073 & & \\
\hline IV & $86(83)$ & 4.7 & Reference & & & \\
\hline \multicolumn{7}{|l|}{ Histology } \\
\hline Adenocarcinoma & 75 (73) & 9.0 & $0.53(0.32-0.90)$ & 0.021 & $0.63(0.37-1.12)$ & 0.114 \\
\hline Non-adenocarcinoma & $28(27)$ & 2.4 & Reference & & Reference & \\
\hline \multicolumn{7}{|l|}{ Chemotherapy" } \\
\hline Yes & $65(63)$ & 13.4 & $0.26(0.16-0.43)$ & $<0.001$ & $0.52(0.26-1.03)$ & 0.063 \\
\hline No & $38(37)$ & 2.0 & Reference & & Reference & \\
\hline \multicolumn{7}{|c|}{ Reason for emergency admission } \\
\hline \multicolumn{7}{|c|}{ Brain metastasis } \\
\hline Yes & $24(23)$ & 15.4 & $0.60(0.33-1.01)$ & 0.055 & & \\
\hline No & $79(77)$ & 4.6 & Reference & & & \\
\hline \multicolumn{7}{|l|}{ Pleural effusion } \\
\hline Yes & $17(16)$ & 8.1 & $1.12(0.55-2.06)$ & 0.738 & & \\
\hline No & $86(84)$ & 6.4 & Reference & & & \\
\hline \multicolumn{7}{|l|}{ Pneumonia } \\
\hline Yes & $15(15)$ & 10.2 & $0.83(0.38-1.58)$ & 0.590 & & \\
\hline No & $88(85)$ & 5.5 & Reference & & & \\
\hline
\end{tabular}

DFEA: diagnosed following an emergency admission; HR: hazard ratio; ECOG: Eastern Cooperative Oncology Group; PS: performance status; EGFR: epidermal growth factor receptor gene. " : $\mathrm{N}=103 ;$ ๆ : Chemotherapy, including definitive chemoradiotherapy. 


\begin{tabular}{|c|c|c|c|}
\hline Patient characteristics & Improvement & No improvement & Univariate $p$-value \\
\hline Subjects $\mathrm{n}$ & 37 & 58 & \\
\hline Age years & & & 0.033 \\
\hline$<75$ & $28(76)$ & $31(53)$ & \\
\hline$\geqslant 75$ & $9(24)$ & $27(47)$ & \\
\hline Sex & & & 0.278 \\
\hline Male & $20(54)$ & $39(67)$ & \\
\hline Female & $17(46)$ & 19 (33) & \\
\hline Smoking status & & & 0.448 \\
\hline Never-smoker & $10(27)$ & $11(19)$ & \\
\hline Current or former smoker & $27(73)$ & $47(81)$ & \\
\hline Histology & & & 0.354 \\
\hline Adenocarcinoma & $8(22)$ & $18(31)$ & \\
\hline Non-adenocarcinoma & $29(78)$ & 40 (69) & \\
\hline ECOG PS at emergency admission & & & 0.041 \\
\hline 2 & $16(43)$ & $13(22)$ & \\
\hline 3 or 4 & $21(57)$ & $45(78)$ & \\
\hline Stage & & & 0.559 \\
\hline III B & $6(16)$ & $7(12)$ & \\
\hline IV & $31(84)$ & 51 (88) & \\
\hline \multicolumn{4}{|l|}{ Reasons for emergency admission } \\
\hline Brain metastasis & 12 (32) & $12(21)$ & 0.231 \\
\hline Pleural effusion & $12(32)$ & $4(7)$ & 0.002 \\
\hline Pneumonia & $3(8)$ & $10(17)$ & 0.239 \\
\hline
\end{tabular}

Data are presented as $n(\%)$, unless otherwise stated. $N=95$. DFEA: diagnosed following an emergency admission; PS: performance status; ECOG: Eastern Cooperative Oncology Group.

good PS $[21,26]$. Together with the results of our study, this indicates that efforts to improve patient PS after admission might also be vital, even for ICU patients. A larger study of DFEA patients who require ICU attention is required to study this hypothesis further.

In patients with advanced lung cancer, a multidisciplinary approach is important as well as supportive care. This study indicated that a multidisciplinary approach might lead to PS improvement in DFEA patients who were admitted because of symptoms associated with brain metastases, which were the main reason for DFEA. According to previous studies of lung cancer patients who required ICU admission, admission to high-volume centres was associated with lower mortality, and this effect may be related to closer collaboration between the experts [19, 21]. For the management of DFEA patients, a multidisciplinary approach is also necessary to improve PS and facilitate patient-centred decision making for most patients. Furthermore, previous studies also demonstrated that this approach had many benefits for patients with advanced lung cancer [27-30]. Therefore, a multidisciplinary approach should be employed for the treatment of advanced lung cancer patients, especially for DFEA patients.

We also investigated factors affecting PS improvement after emergency admission in DFEA patients. 37 (39\%) DFEA patients showed improved PS after admission and the predictive factors for PS improvement were younger age, PS of 2, and emergency admission due to pleural effusion. These results indicate that particular attention should be paid to DFEA patients with these features.

PS was the most important factor for evaluating the appropriateness of chemotherapy. The benefits of chemotherapy in patients with poor PS are speculative, and many oncologists only consider chemotherapy appropriate for patients with an ECOG PS of 2 [31-35]. However, the results of the present study suggest that non-chemotherapeutic supportive care can improve the PS in DFEA patients and that PS after supportive care is a better predictor of overall survival than PS at emergency admission. The appropriateness of chemotherapy should, therefore, not be assessed on the basis of PS at emergency admission, and maximum supportive care should be applied in all patients.

This study had some limitations. First, there was the potential for selection bias, because this study was conducted at a single institution and our hospital plays a central role in treating emergency patients in the surrounding area. Secondly, we were unable to distinguish fully between patients with disease-related poor PS and those with long-standing poor PS. Although the PS improved in some patients after chemotherapy, 
we were unable to analyse the effects of improvements in PS after chemotherapy. Thirdly, the duration of supportive care before chemotherapy was determined by each doctor and, therefore, differed among patients.

In conclusion, DFEA is relatively common. Although the survival of DFEA patients is poor, DFEA was not an independent predictor of overall survival according to multivariate analysis. Additionally, good PS just prior to opting for chemotherapy, but not at emergency admission, was the only independent predictor of longer overall survival in DFEA patients. Efforts to improve patient PS after admission should, thus, be considered vital in such circumstances.

\section{Acknowledgements}

The authors would like to thank Keiko Sakuragawa (Dept of Respiratory Medicine, Kobe City Medical Center General Hospital, Kobe, Japan) for her administrative assistance.

\section{References}

Siegel R, Naishadham D, Jemal A. Cancer statistics, 2013. CA Cancer J Clin 2013; 63: 11-30.

2 Ramalingam SS, Owonikoko TK, Khuri FR. Lung cancer: new biological insights and recent therapeutic advances. CA Cancer J Clin 2011; 61: 91-112.

3 Elliss-Brookes L, McPhail S, Ives A, et al. Routes to diagnosis for cancer - determining the patient journey using multiple routine data sets. Br J Cancer 2012; 107: 1220-1226.

4 Public Health England. National Cancer Intelligence Network. www.ncin.org.uk/home Date last accessed: November 1, 2013

5 Morgan HK, Hodgson L, Baldock E, et al. P195 Outcomes in emergency admissions with lung cancer: a 1-year perspective from a teaching hospital. Thorax 2011; 66: A146-A147.

6 Beckett P, Tata LJ, Hubbard RB. Risk factors and survival outcome for non-elective referral in non-small cell lung cancer patients - Analysis based on the National Lung Cancer Audit. Lung Cancer 2014; 83: 396-400.

7 Yamaguchi S, Ohguri T, Matsuki Y, et al. Palliative radiotherapy in patients with a poor performance status: the palliative effect is correlated with prolongation of the survival time. Radiat Oncol 2013; 8: 166.

8 Sabur NF, Chee A, Stather DR, et al. The impact of tunneled pleural catheters on the quality of life of patients with malignant pleural effusions. Respiration 2013; 85: 36-42.

9 Fujimoto D, Ueda H, Shimizu R, et al. Features and prognostic impact of distant metastasis in patients with stage IV lung adenocarcinoma harboring EGFR mutations: importance of bone metastasis. Clin Exp Metastasis 2014; 31: 543-551.

10 Goldstraw P, Crowley J, Chansky K, et al. The IASLC Lung Cancer Staging Project: proposals for the revision of the TNM stage groupings in the forthcoming (seventh) edition of the TNM Classification of malignant tumours. $J$ Thorac Oncol 2007; 2: 706-714.

11 Nagai Y, Miyazawa HHuqun et al. Genetic heterogeneity of the epidermal growth factor receptor in non-small cell lung cancer cell lines revealed by a rapid and sensitive detection system, the peptide nucleic acid-locked nucleic acid PCR clamp. Cancer Res 2005; 65: 7276-7282.

12 Blum TG, Rich A, Baldwin D, et al. The European initiative for quality management in lung cancer care. Eur Respir J 2014; 43: 1254-1277.

13 Gorham J, Ameye L, Berghmans T, et al. The lung cancer patient at the emergency department: a three-year retrospective study. Lung Cancer 2013; 80: 203-208.

14 Mayer DK, Travers D, Wyss A, et al. Why do patients with cancer visit emergency departments? Results of a 2008 population study in North Carolina. J Clin Oncol 2011; 29: 2683-2688.

15 Kotajima F, Kobayashi K, Sakaguchi $\mathrm{H}$, et al. Lung cancer patients frequently visit the emergency room for cancer-related and -unrelated issues. Mol Clin Oncol 2014; 2: 322-326.

16 Meert AP, Sculier JP. Aetiological diagnosis of acute respiratory failure in critical care oncology. Eur Respir J 2013; 42: 299-301.

17 Berghmans T, Pasleau F, Paesmans M, et al. Surrogate markers predicting overall survival for lung cancer: ELCWP recommendations. Eur Respir J 2012; 39: 9-28.

18 Neal RD, Allgar VL, Ali N, et al. Stage, survival and delays in lung, colorectal, prostate and ovarian cancer: comparison between diagnostic routes. Br J Gen Pract 2007; 57: 212-219.

19 Slatore CG, Cecere LM, Letourneau JL, et al. Intensive care unit outcomes among patients with lung cancer in the surveillance, epidemiology, and end results-medicare registry. J Clin Oncol 2012; 30: 1686-1691.

20 Bonomi MR, Smith CB, Mhango G, et al. Outcomes of elderly patients with stage IIIB-IV non-small cell lung cancer admitted to the intensive care unit. Lung Cancer 2012; 77: 600-604.

21 Soares M, Toffart AC, Timsit JF, et al. Intensive care in patients with lung cancer: a multinational study. Ann Oncol 2014; 25: 1829-1835.

22 Soares M, Darmon M, Salluh JI, et al. Prognosis of lung cancer patients with life-threatening complications. Chest 2007; 131: 840-846.

23 Soares M, Caruso P, Silva E, et al. Characteristics and outcomes of patients with cancer requiring admission to intensive care units: a prospective multicenter study. Crit Care Med 2010; 38: 9-15.

24 Adam AK, Soubani AO. Outcome and prognostic factors of lung cancer patients admitted to the medical intensive care unit. Eur Respir J 2008; 31: 47-53.

25 Azevedo LC, Caruso P, Silva UV, et al. Outcomes for patients with cancer admitted to the ICU requiring ventilatory support: results from a prospective multicenter study. Chest 2014; 146: 257-266.

26 Toffart AC, Minet C, Raynard B, et al. Use of intensive care in patients with nonresectable lung cancer. Chest 2011; 139: 101-108.

27 Ellis PM. The importance of multidisciplinary team management of patients with non-small-cell lung cancer. Curr Oncol 2012; 19: S7-S15. 
28 Jung H, Sinnarajah A, Enns B, et al. Managing brain metastases patients with and without radiotherapy: initial lessons from a team-based consult service through a multidisciplinary integrated palliative oncology clinic. Support Care Cancer 2013; 21: 3379-3386.

29 Kelley AS, Meier DE. Palliative care - a shifting paradigm. N Engl J Med 2010; 363: 781-782.

30 Temel JS, Greer JA, Muzikansky A, et al. Early palliative care for patients with metastatic non-small-cell lung cancer. N Engl J Med 2010; 363: 733-742.

31 Lilenbaum RC. Treatment of advanced non-small-cell lung cancer in special populations. Oncology (Williston Park) 2004; 18: 1321-1325.

32 Azzoli CG, Baker S Jr, Temin S, et al. American Society of Clinical Oncology Clinical Practice Guideline update on chemotherapy for stage IV non-small-cell lung cancer. J Clin Oncol 2009; 27: 6251-6266.

33 Zukin M, Barrios CH, Pereira JR, et al. Randomized phase III trial of single-agent pemetrexed versus carboplatin and pemetrexed in patients with advanced non-small-cell lung cancer and Eastern Cooperative Oncology Group performance status of 2. J Clin Oncol 2013; 31: 2849-2853.

34 Helbekkmo N, Strøm HH, Sundstrøm SH, et al. Chemotherapy and quality of life in NSCLC PS 2 patients. Acta Oncol 2009; 48: 1019-1025.

35 Morth C, Valachis A. Single-agent versus combination chemotherapy as first-line treatment for patients with advanced non-small cell lung cancer and performance status 2: a literature-based meta-analysis of randomized studies. Lung Cancer 2014; 84: 209-214. 